\title{
Hybrid WSN and RFID indoor positioning and tracking system
}

\author{
Zhoubing Xiong $^{1 *}$, Zhenyu Song ${ }^{1}$, Andrea Scalera ${ }^{2 *}$, Enrico Ferrera ${ }^{2}$, Francesco Sottile ${ }^{2}$, Paolo Brizzi ${ }^{2}$, \\ Riccardo Tomasi $^{2}$ and Maurizio A Spirito ${ }^{2}$
}

\begin{abstract}
Wireless sensor networks (WSNs), consisting of a large number of nodes to detect ambient environment, are widely deployed in a predefined area to provide more sophisticated sensing, communication, and processing capabilities, especially concerning the maintenance when hundreds or thousands of nodes are required to be deployed over wide areas at the same time. Radio frequency identification (RFID) technology, by reading the low-cost passive tags installed on objects or people, has been widely adopted in the tracing and tracking industry and can support an accurate positioning within a limited distance. Joint utilization of WSN and RFID technologies is attracting increasing attention within the Internet of Things (IoT) community, due to the potential of providing pervasive context-aware applications with advantages from both fields. WSN-RFID convergence is considered especially promising in context-aware systems with indoor positioning capabilities, where data from deployed WSN and RFID systems can be opportunistically exploited to refine and enhance the collected data with position information. In this paper ${ }^{a}$, we design and evaluate a hybrid system which combines WSN and RFID technologies to provide an indoor positioning service with the capability of feeding position information into a general-purpose loT environment. Performance of the proposed system is evaluated by means of simulations and a small-scale experimental set-up. The performed analysis demonstrates that the joint use of heterogeneous technologies can increase the robustness and the accuracy of the indoor positioning systems.
\end{abstract}

\section{Introduction}

Recent technological developments in the miniaturization of electronics and wireless communication technology have motivated the development of small-sized, low-power, and inexpensive sensing and radio-equipped devices, and dramatically reduce the cost of deploying pervasive monitoring and tracking applications in large-scale scenarios where various data are collected from hundreds of different locations. Since it has been practical in the last few years to collect, process, and exploit massive data from millions or even billions of devices, new paradigms are emerging based on the global Internet of Things (IoT) to extend the border of the current Internet to the physical world. The IoT makes every physical object become a potential part of a distributed network in which heterogeneous devices autonomously and spontaneously

\footnotetext{
*Correspondence: zhoubing.xiong@polito.it; scalera@ismb.it

1 Department of Electronics and Telecommunications, Politecnico di Torino,

Torino, Italy

${ }^{2}$ Pervasive Technologies, Istituto Superiore Mario Boella, Torino, Italy
}

abstract and share context information from the real world [1].

Unlike the traditional pervasive systems, which are specifically designed to monitor a predefined set of interested physical phenomena, e.g., vibration for structural health monitoring systems and temperature for energy optimization systems, the IoT conceives a single pervasive network to support seamless, interoperable, crossapplication data collection from any kind of device for any type of information. In addition to the data collection procedures, establishment of the relationship among the collected samples is commonly recognized as another significant issue in such a scenario, i.e., constructing the 'context' information [2]. In IoT environments, position information covers a primal role because it provides useful context knowledge to be associated with other monitored parameters. For example, the meaning of a temperature reading could vary significantly in case it is close to a window, or on top of an heater, etc.

\section{算 Springer}

(c) 2013 Xiong et al: licensee Springer. This is an Open Access article distributed under the terms of the Creative Commons Attribution License (http://creativecommons.org/licenses/by/2.0), which permits unrestricted use, distribution, and reproduction in any medium, provided the original work is properly cited. 
Wireless sensor networks (WSNs) represent a key technology for IoT scenarios (such as environmental monitoring, e-health, surveillance, and manufacturing [3]). A WSN is a community of objects, where those objects are usually small-embedded devices with capability of sensing physical phenomena in the environment and are equipped with radio components to communicate with each other wirelessly. Through their on-board radio interfaces, these devices can collect or disseminate data and collaboratively form a cooperative network either $a d$ hoc or with a clusterbased architecture to perform some specific actions in large-scale static or mobile environments [4]. WSN nodes leverage on a common set of protocols and algorithms to set-up an ad hoc network to transport data in multi-hop fashion to one or more central nodes namely 'sink nodes' or gateways, which in turn provide connectivity towards the Internet. As an innovative and powerful solution for various kinds of applications, WSN is especially suitable to be adopted when the range of the monitored area exceeds a single device's radio range, when cost-effective monitoring is required and/or simple in-network processing of physical parameter is needed.

By associating a unique digital identifier with each physical item, radio frequency identification (RFID) technology becomes a fundamental technique in the IoT scenario [5]. The confluence of the absence of batteries, the low cost, and the rapid proliferation of passive RFID tags in the past decade have made the RFID technology revolutionize the tracing and tracking industry and become a de facto reference technology [6].

Although WSN and RFID can be considered as substitutive technologies in some use cases, they are historically born to cover different needs. Recently, a number of researchers are endeavoring to jointly utilize these technologies to exploit the advantages of both systems [7-9].

This work evaluates how the joint use of RFID and WSN technologies can be effectively exploited within IoT positioning and tracking systems. The motivating goal of this research is to provide a whole range of location-based service with more precise and more reliable results according to various needs. To achieve this goal, the diverse data collected by WSN nodes are associated with a wider set of position information which are allowed to be further exploited by context-aware systems.

According to the work in [10], the locating systems can be preliminarily grouped into two major categories: receptive locating systems and transmissive locating systems. In the receptive locating systems, the position information is distributed ubiquitously, and the mobile device can derive its own location from this information [the global positioning system (GPS) is the most representative example]. The mobile device can independently locate the derived position in a map, so a local service (without revealing its position to a third party) can be easily provided on top of this locating information, or some value-added services can be obtained by sharing this locating information with others. Conversely, in the transmissive locating systems, the position is derived by a fixed station which either sees the mobile device or receives a beacon from it. The station can transmit the derived locating result back to the device or use it to generate other value-added services. Subcell global system for mobile communications or GSM positioning is a prime example employs this approach, which leverages the mobile communication channel beacon. Also hybrid techniques combining these principles are possible, and this paper will actually underline this possibility.

Despite years of research and experimentation, very few positioning technologies, apart the GPS, have nowadays a significant economical impact. Only a few sets of technology are available for indoor locating, usually designed for niche or legacy markets. The reasons of this poor diffusion include high costs compared to the added value achieved, technology constraints (regarding precision, reliability, and performances), and, generally speaking, the lack of killer applications.

Starting from previous works where the feasibility of the joint use of WSN and RFID in indoor positioning applications was assessed through simulations [11,12], this paper outlines the reference design of a hybrid indoor positioning system leveraging both WSN radio information and RFID detection events. Parts of this paper was presented in our previous work in [13], which provided the initial glance of the hybrid WSN-RFID localization system. First, this paper goes into more details of the proposed hybrid reference architecture. Second, it details the formulation of the designed hybrid positioning algorithm and provides an analysis of the related computational complexity. Third, it compares the performance of different variants of the same hybrid approach. Forth, it calibrates the received signal strength indicator (RSSI) model on the basis of real experimental measurements. Finally, it improves the performance of the localization algorithm by introducing some new robustness conditions based on the WSN and RFID ranging models. In particular, the performance is evaluated by means of both computer simulations and through a small-scale experimental set-up.

The remainder of the paper is organized as follows: Section 2 provides a brief overview of the state of the art of indoor positioning systems. Then the reference architecture and structural components (namely the WSN and the RFID segment) of the proposed solution are elaborated together with the field trial scenario in Section 3. The positioning algorithm employed by the system is deeply analyzed and illustrated in Section 4. Furthermore, the system is validated in the controlled conditions, and the simulation and experimental results are presented in the Section 5. In Section 6 we draw the conclusions. 


\section{Related work}

Positioning systems generate a lot of interest and effort both in academic and industrial research and nowadays, a lot of technologies can be used and mixed (e.g., ultrasound, laser scanner, infrared, camera vision, radio frequencies, and custom sensors). Each system has addressed the aggregation of sensor data into location estimations via most suitable methods.

Positioning and tracking are crucial features in many ubiquitous computing and robotics applications where knowledge about the location of the entities (i.e., people and objects) is required $[14,15]$.

Nowadays, most widely advanced positioning services have been thought for outdoor scenarios. Indeed, radar locating systems for ships and aircrafts are used for the longest time for historical reasons. Global navigation satellite systems (GNSS) - such as GPS and Galileo - are mature technologies for vehicle navigation and are widely adopted in everyday life. Unfortunately, both these technologies are not suitable for indoor environments, the first because of the severity of multipath noise from which it is afflicted and the difficulties of multi-object localization, the second because of the buildings obstructiveness on GNSS signals.

In order to achieve people and object localizations in indoor environments by overcoming such disadvantages, significant research has been conducted over the years in different indoor positioning systems (IPSs) [16-18].

Dempsey [19] defines an IPS as a system which can infer the position of a target inside the physical space where the detection system is installed, within a maximum time delay or in real time. In the second case, it is usual to speak of real-time locating system (RTLS), which, standing to an ISO definition [20], is the ability to locate the position of an item anywhere in a defined space at a point in time that is, or is close to, real time. Generally speaking, the RTLS definition is used when discussing about asset locating and about products or goods tracking and traceability.

IPSs are based on some prior knowledge about position of special nodes, namely the anchor nodes, and aim at estimating position of one or more mobile nodes, whose positions are unknown, by processing ranging data collected and exchanged by both mobile and anchor nodes.

According to Liu [21], there are four different system topologies for IPSs: (1) remote positioning system, (2) self-positioning systems, (3) indirect remote positioning systems, and (4) indirect self-positioning systems. In remote positioning system, a mobile node acts as main signal transmitter and several anchor measuring units receive and measure its broadcasted signal. The results from all measuring units are collected, and the location of the transmitter is computed in a central master station. In self-positioning systems, the mobile acts instead as measuring unit. This unit receives the signals of several transmitters in known locations and computes its location locally based on the measured signals. Two middle-way approaches are also possible: in indirect remote positioning systems, measurements collected by the mobile node are transmitted via a wireless data link for remote position computation; in indirect self-positioning systems, measurements collected locally by fixed stations are transmitted to the mobile through a wireless data link.

IPSs can also be classified according to the employed position estimation technique. Different positioning techniques can be combined to compensate the limitations of a single method.

Angle of arrival or AoA method is based on the receiver antenna amplitude or phase response. The accuracy of this method depends on the antenna directivity, multipath reflection, and signal shadowing; overall they can achieve 2 to $4 \mathrm{~m}$ accuracy [22]. It presents two main problems: nodes require a directional antenna with beam forming and line of sight propagation path is needed between the transmitter and the receiver.

Time of arrival (ToA) and time difference of arrival or TDoA methods are both based on measurement of the propagation time. These methods are hard to implement in radio frequency IPSs because very accurate timers are needed to reach an acceptable accuracy. Furthermore, within environments affected by multipath, the detection of time of arrival is accurate only for very large signal bandwidths. For this reason, some systems use ultra-wide band (UWB) technology for an accurate ToA estimation. The proximity method, also known as cell of origin $(\mathrm{CoO})$, consists in detecting an entity presence inside a limited area, or cell, in which coordinates are known.

RSSI method is instead based on the measurement of radio power at the receiver. Despite the fact that RSSI measurement is time varying and unstable under most circumstances, RSSI-based solutions are widely used as localization technique in WSN systems. RSSI measurements are in fact adopted in many wireless sensor network (WSN) communication standards and are thus made available at no cost by normal radio transceivers installed on-board WSN nodes, without need for additional hardware affecting power consumption and size or cost of WSN nodes.

Two common techniques to exploit RSSI for localization are based on fingerprinting signal strengths and conversion of signal strength to distance. In fingerprinting techniques, a map of the signal strength behavior in the coverage area is constructed. In a first phase, a set of offline measurements is performed to build a database; then, during the real-time location phase, the algorithm searches for the best matches between the RSSI samples and the stored values. Precision of such methods is normally limited: MoteTrack [23] can achieve an $80 \%$ location-tracking accuracy of $1.6 \mathrm{~m}$ and Ekahau positioning engine [24] 
achieves an accuracy of 1 to $2 \mathrm{~m}$. The disadvantage of this method is the tiresome calibration phase, during which large amounts of measurements are collected to construct the database. Furthermore, if prior measurements are used when an environmental change occurs, a new calibration phase is needed.

Another family of techniques involves conversion of RSSI to distance using Friis equation [25]. This equation establishes the strength of a signal sent by a radio transmitter in free space at one particular distance, following an exponential relation. In this kind of algorithms, transmitting nodes (either anchors or mobiles) broadcast their last known position along with any RSSI information previously collected from other nodes. Using the exponential relation, the receiver can convert the RSSI measurements into distances and, using triangulation, estimate its location in relation to the anchors. Locating errors using these methods are in average slightly higher than in fingerprinting due to two main reasons: the way that the empirical RSSI-distance relation differs from the theoretical model assumed in the algorithm and the environmental changes affecting RSSI stability. Although this kind of algorithms provide lower accuracy than other techniques, their simplicity makes them more suitable to be employed in low-power systems.

Finally, IPSs can be classified on the different underlying technologies adopted for ranging. In the field of those working at radio frequency, each adopted technology brings unique advantages and disadvantages in the indoor position inference. Wireless local area network (WLAN) technology $[17,26]$ is widespread; and all types of mobile device, from laptop to smartphone and tablet, are nowadays able to communicate with this standard. WLAN can be used to estimate the location of a mobile device within the local network without line-of-sight necessity. Most positioning methods in WLAN locating systems are based on RSSI. The accuracy obtained using this technology ranges from meters to tens of meters. Room-level accuracy can be reached using Bluetooth [27-30]. The major issue using this technology is the unsuitability in RTLS applications because of the delay caused by the inquiry scan process that is performed by a device to detect other devices. UWB is a short-range and high-bandwidth communication technology, with strong multipath resistance and building penetrability. UWB has recently gained a lot of interest in indoor positioning researches thanks to its theoretical accuracy that is in the order of few centimeters [31-34]. An issue of UWB regards the expensive cost of a single node which makes the technology unsuitable for extensive deployments.

In the following subsections, two technologies will be introduced which have been used for the implementation of the hybrid positioning system explained in this paper: WSN based on IEEE 802.15.4 standard and RFID.

\subsection{IEEE 802.15.4-based WSN positioning systems}

IEEE 802.15.4 is a standard which specifies the physical and media access control layers for a low-power and lowrate wireless personal area networks (PANs). It is the basis for a number of specifications, such as ZigBee, which further extend the standard by developing the upper layers which are not defined in IEEE 802.15.4.

ZigBee nodes can communicate each other within a range of nearly $100 \mathrm{~m}$ outdoors, in free space, but indoors it is usually 5 to $20 \mathrm{~m}$. To determine the distance between two nodes, RSSI technique is typically adopted. ZigBee is particularly affected by service interruptions which is also due to the band frequency in which it communicates, and its band is also occupied by noisy communication protocols such as WiFi and Bluetooth. ZigBee is particularly affected by service interruptions which are due to the overlapping of its operating frequency band with noisy communications protocols such as WiFi and Bluetooth.

Tadakamadla [35] deployed ZigBee network for monitoring the presence and movements of vehicles and humans into an indoor environment. It uses the RSSI to determine the position of tagged entities; the randomness of RSSI and the dependency on the user's body and orientation cause the main error contribution. In this work an accuracy of $3 \mathrm{~m}$ and $35 \%$ precision were obtained.

Larranaga et al. [36] used ZigBee network to monitor an area of $432 \mathrm{~m}^{2}$. The network consists of eight reference nodes, and RSSI is used to locate mobile nodes. In this work an average localization accuracy of $3 \mathrm{~m}$ was obtained.

My Bodyguard [37] is a commercial system that tracks objects and people. It is based on the ZigBee for indoor environments and on GNSS and cellular networks for outdoor environments. With this device a room-level accuracy is obtained.

Alternatively, IEEE 802.15.4 can also be used with 6LoWPAN and standard Internet protocols to build a wireless embedded Internet. The WSN used for the IPS developed for this work is based on 6LoWPAN.

\subsection{RFID positioning systems}

RFID is a technology that allows to identify an object, called tag, and reading the unique code stored within tag itself. A typical RFID system is made by at least three components: the radio frequency transponder, the reader (a transceiver controlled by a microprocessor used to inquiry a tag), a client software (communicate with a reader through a reader protocol, collecting, storing, and/or processing codes retrieved from the tags). In RFID-based positioning systems, $\mathrm{CoO}$ positioning method is principally used. Using these positioning methods, the accuracy is highly dependent on the number of tags involved and on the maximal reading range. RSSI is used for applying multilateration positioning method. Povalac and Sandebesta 
[38], Nikitin et al. [39], and Arnitz et al. [40] analyzed both phases of arrival positioning methods for RFID-based locating systems.

Because of the characteristics of electromagnetic waves to penetrate solids, RFID-based locating systems have the ability to detect a tag even without direct line of sight (without metal or water). Thanks to this characteristics, it is possible to embed tags into the wall, ceiling, or pavement of a building, providing almost completely unobtrusive systems. Some scalability issues can rise when a large number of tags and readers are used: a complex system configuration and management is required. In active RFID system, the readers communicate with active tags equipped with internal batteries. Active tags are more expensive than passive tags but allow a longer communication range (tens of meters). Passive RFID tags have the advantage of the small size, high level of ruggedness, relatively inexpensive installation, and low maintenance needs; the theoretical detection range is within $10 \mathrm{~m}$ but the reflections can cause false readings which heavily affect the effectiveness of the localization. IPSs based on RFID systems have been widely explored and discussed in scientific literature [41-46]. While in WSN-based IPSs, anchor and mobile nodes are normally realized using the same hardware and exchange ranging information in a peer-to-peer fashion; in RFID-based IPSs, two distinct schemes are instead generally possible [44]: (1) in the 'active' scheme, the mobile node is implemented by a portable RFID reader, while tags are used as anchors; (2) in the 'passive' scheme, RFID tags are instead objects to be located while RFID readers are in known position. While the choice of the scheme to be applied depends on application requirements (e.g., the number of objects to locate, etc.), both schemes can be used with different types of tags (e.g., HF/UHF tags, active/passive tags, etc.), providing different performance in terms of maximum range (from a few centimeters to $10 \mathrm{~m}$ ), propagation model, and costs [45]. Seco et al. [47] deployed a system that use nearly 70 active tags scattered into 55 rooms and covering 1,600 $\mathrm{m}^{2}$ area. Using RSSI method in this work. a $1.5-\mathrm{m}$ accuracy is obtained. Kimaldi [48] provides commercial systems for hospitals in locating application deployment. Personnel monitoring and access control have been obtained using wristbands and keyring tags. Daly et al. [49] deployed a passive RFID-based positioning system which has been embedded with passive RFID tags in pavement for navigation purpose. Kiers et al. [50] deployed a navigation system using arrays of passive RFID tags which have been installed under a carpet to provide path indication to blind people. Peng et al. [51] deployed an hybrid system composed by active RFID system and GNSS in order to make a positioning system that works seamlessly outdoor and indoor. By using Kalman filters in this work, a meter accuracy is obtained.
Table 1 Typical accuracy and positioning methods of radio frequency positioning technologies

\begin{tabular}{lll}
\hline Technology & Accuracy & Positioning methods \\
\hline WLAN & Meters & Fingerprinting and CoO \\
Bluetooth & Decimeters to meters & Fingerprinting and CoO \\
ZigBee & Meters & RSSI \\
RFID & Decimeters to meters & Fingerprinting and CoO \\
UWB & Centimeters to decimeters & ToA \\
\hline
\end{tabular}

Table 1 shows the typical values of accuracy and positioning methods used in radio frequency IPSs.

IPSs can use single location technology or the combination of multiple technologies together in hybrid systems to increase both positioning accuracy and system robustness.

\section{Reference architecture and design}

The proposed positioning system combines WSN and RFID in order to compensate the limitations of each technology. On one hand, the WSN provides a good radio coverage but with a low positioning accuracy due to the high noise on RSSI measurements. On the other hand, the RFID technology provides the following: (1) in the case of high-frequency (HF), very precise positioning information but limited coverage and temporal discontinuity; (2) in the case of ultra-high frequency (UHF), good coverage and reliability but high granularity of the location. The appropriate combinations of the two technologies could be a good strategy in building indoor positioning and tracking system with increased positioning accuracy and availability.

Figure 1 presents the hybrid architecture of the hybrid positioning system, and the field data are collected by two different systems, WSN segment and RFID segment.

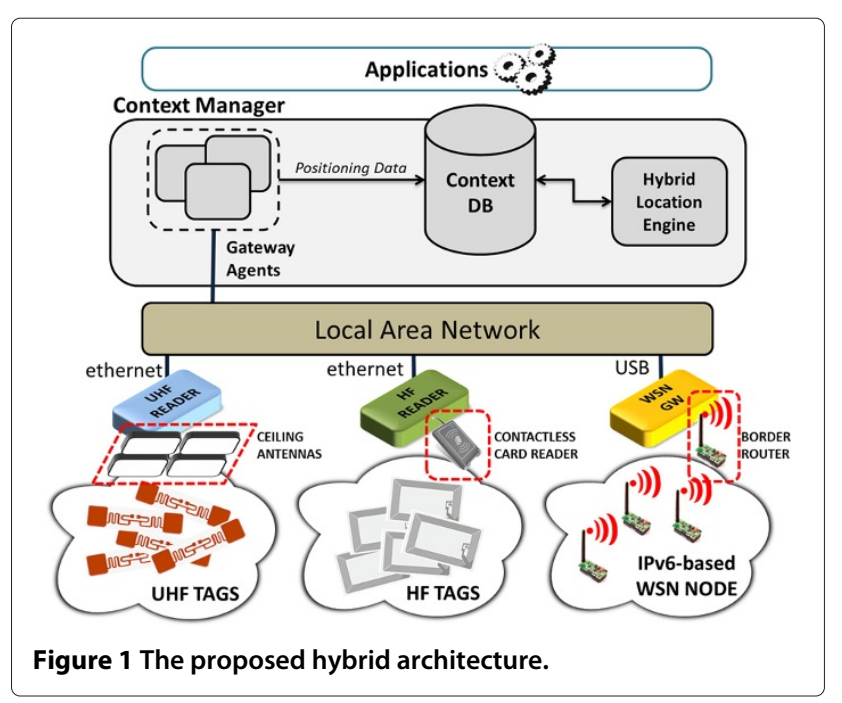




\subsection{WSN segment}

The WSN segment is a self-configuring, IPv6-based sensor network which have been implemented and tested on Telos rev.B [52] and STM32W [53] nodes as shown in Figure 2. On the software side, each node runs a Contiki operating system [54]; on the hardware side, each node is equipped with a radio transceiver, a microcontroller (TI MSP430 for Telos rev.B and ARM Cortex-M3 for STM32W; Moteiv Corporation, El Cerrito, CA, USA) and some on-board sensors (e.g., button sensors, temperature sensors and light sensors).

In the network level, out-of-band control messages are exchanged among the nodes to help each node to build its neighbor list and autonomously form the network. Each node periodically updates its neighbor list and dynamically builds an optimal route to every potential destination.

Within the WSN segment, the positioning data are collected in the following process:

1. The distances between the mobile node (node to be located) and other nodes (two anchor nodes and/or any other possible mobile nodes) are measured in terms of the RSSI.

2. The measured RSSI values by the mobile node (node to be located) are directly sent to a fixed infrastructure, or forwarded by the router nodes (could be an anchor node or a mobile node), to a fixed infrastructure when a multi-hop transmission is required.

3. After being processed by the positioning algorithm that is running on the fixed infrastructure, the locating result is sent back to the requester (the mobile to be located).

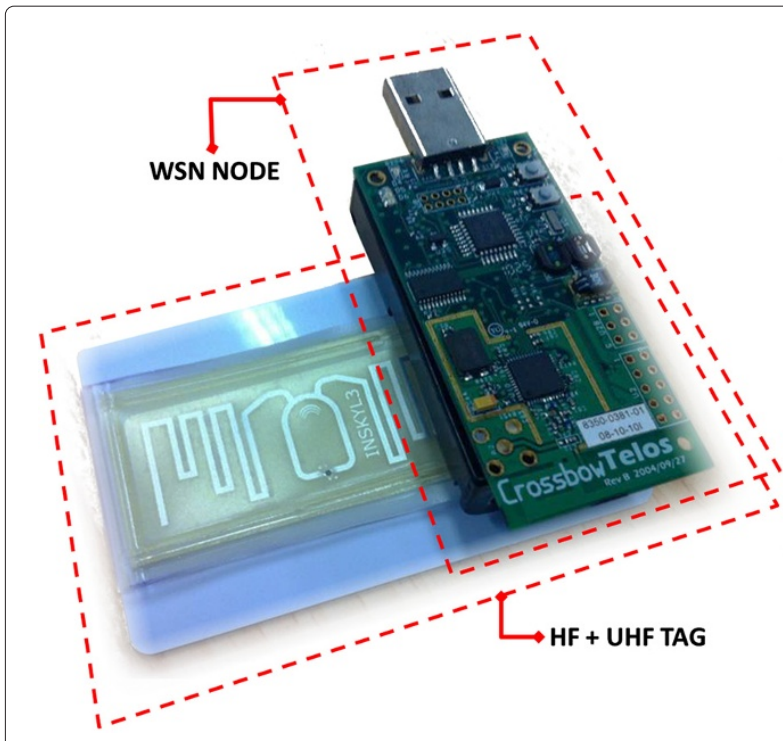

Figure 2 Multi-technology node used for tests.
In terms of communication, a WSN segment is divided into three levels:

- The main gateway, also called the concentrator

- The fixed gateway

- The network nodes

In order to obtain the RSSI information, each mobile node periodically broadcasts a user datagram protocol or UDP ranging request, which is used by neighbor nodes to measure uplink RSSI. Anchor nodes reply in turn with a ranging response, including the measured uplink RSSI. Finally, the mobile node measures all downlink RSSI, aggregates all ranging responses, and forwards all the uplink-downlink tuples (one for each neighbor) to the WSN gateway. The WSN gateway is a simple commercial off-the-shelf (COTS) low-power PC running Linux (Vancouver, Canada).

\subsection{RFID segment}

The RFID segment is composed of two systems, a UHFRFID system and an HF RFID system. They are independent from each other and provide separate detection for the RFID tags.

In the HF RFID system, some contactless badge readers are placed at the room entrances, and they produce positioning information while the user register (or request access) his passage through a door. This information is extremely accurate, but could instantly lose value even over a short period of time - when the user enters or exits a room - if not fused with other information. The UHF system is composed of a set of RFID reader plus four compliant antennas deployed on the ceiling. When a UHF tag is under, Figure 3 depicts the test-bed scenario while Figure 4 provides a snapshot of the actual deployment (within ISMB labs). The typical 4-antennas/reader combination has been used, in order to simplify the field trial; however, a more complex antennas multiplexing should be used in an hypothetical wider deployment (at least

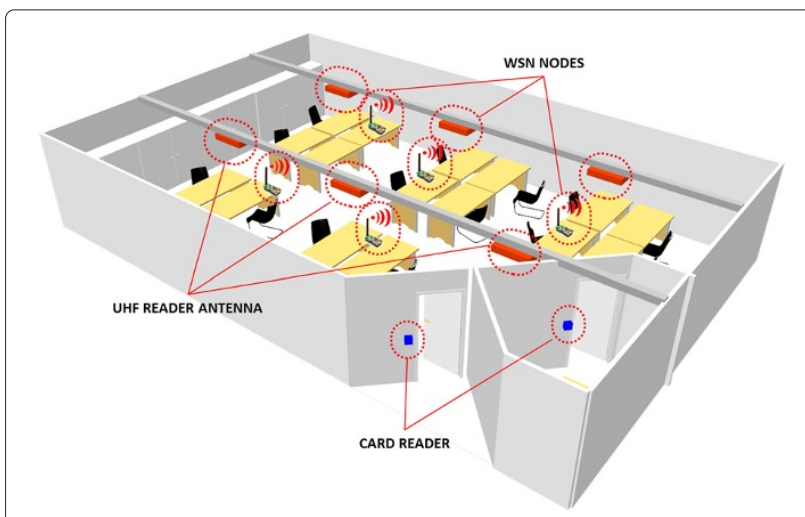

Figure 3 RFID-enhanced WSN positioning system schema. 


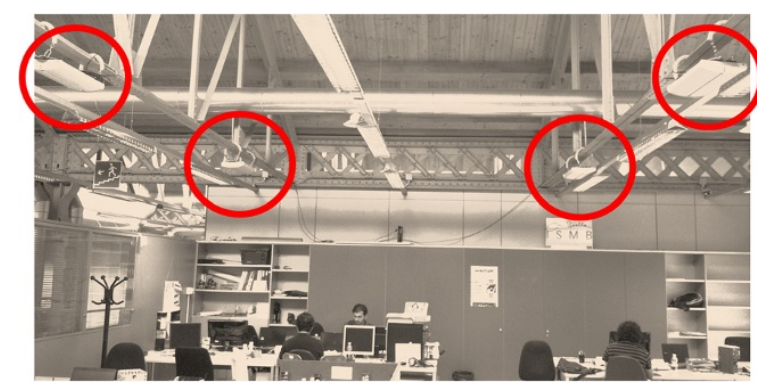

Figure 4 RFID antennas on the ceiling.

32 antennas/reader). The physical attributes, the relative position, and the power irradiation level of each antennas has been chosen to optimize the coverage area, trying to avoid the overlap of each antenna coverage range. Of course it is impossible to avoid reading the same tag by different antennas: for this reason, it has been used as a simple algorithm based on the number of readings of a single antenna, in order to univocally associate a position (as a 'zone').

The RFID segment is based on a set of HF COTS and UHF-RFID readers which irradiate periodically and issue an event when a new tag is detected.

Data collected by the two segments are preprocessed by technology-specific gateways and then transferred through local area network to a central entity named context manager, which is a virtually distributed entity capable of handling generalized context information extracted from different platform-specific components. Within the context manager, a virtual delegate named gateway agent is configured to filter all the data from the specific gateway and feed them into any subscribing entity, e.g., a system which is interested in receiving these specific data. Based on such data and configuration data hosted inside the context manager, the location engine (described in Section 3.3) is able to extract the physical location of objects (RFID tags and WSN nodes) associated with the sources of the physical-world events.

Since different types of technology are adopted, the proposed system is classified as a hybrid scheme exploiting both indirect remote positioning systems and indirect self-positioning. Hence, the location engine is named as hybrid location engine.

\subsection{Hybrid location engine}

The hybrid location engine is the core of the positioning and tracking system. As it can be seen from Figure 1, it is a centralized location engine where a hybrid positioning algorithm is implemented to periodically estimate the positions of all the unknown mobile nodes. As shown in Figure 2, a typical mobile node is equipped with three radio frequency (RF) devices: a WSN node, a UHF-RFID tag, and an HF badge. Moreover, the system allows the existence of other combination of RF devices: two of the three different elements (e.g., a WSN node and an HF badge) or just with single device (e.g., a WSN node or a UHF-RFID tag).

As indicated in Figure 1, three different observations (RSSI measurements derived from WSN nodes, detection of events from UHF-RFID tags, and HF badges) are sent to a context data base (DB). Since the UHF-RFID and HFRFID detection events are available at the corresponding readers, these data are not forwarded to the corresponding unknown mobile nodes, for instance, through the WSN technologies, to implement a distributed positioning algorithm. On the contrary, in order to reduce communication latency and network traffic, all data, including also RSSI measurements from WSN devices, are collected in the $\mathrm{DB}$, then the hybrid location engine estimates the position of the mobile nodes in a centralized way.

The main task of the hybrid location engine is to estimate the positions of mobiles. But some other tasks, for instance, reading location information of anchors and measurements for mobile nodes, are done to accomplish this task. In every $\Delta \mathrm{T}_{p}$ seconds, it completes the following processes:

1. Location information reading. At the beginning of each time step $\Delta \mathrm{T}_{p}$, the hybrid location engine queries the DB about the location information for all the devices. In more detail, the location information includes the unique device ID and the corresponding device category (e.g., WSN, UHF RFID, or HF RFID). For simplicity, the device ID is a five-digit number and is general for all the device. For each device there is a flag which indicates if it is fixed or mobile. A fixed device may be either a WSN anchor, a UHF-RFID antenna, or an HF badge reader whose positions are perfectly known and are stored in the DB; while a mobile device is a movable node whose position is not known. In addition, the device association information is read. As mentioned above, a mobile node may be equipped with different RF devices and the association information specifies how different RF devices are bound with together. The association information is useful, since in the DB an observation (a RSSI measurement and a detection of UHF tag or HF badge) is only related to a single RF device. Please note that this information reading step is performed at each $\Delta \mathrm{T}_{p}$, because the network topology may change with time, for example, node changing (e.g., a new node joins the network, a node leaves, or the known position changes), association changing (e.g., new devices are bound together or the old association changes), or role changing (e.g., a mobile node becomes an anchor node or an anchor 
node becomes a mobile node). By doing this, the location engine is able to follow the latest change of network topology and to have the capability of good position estimates.

2. Measurements reading. During this step, the hybrid location engine reads all the available observations from the DB. These observations could be RSSI, UHF-RFID tag, and HF badge detection events, and the time interval is chosen from some previous time to current time $t_{k}$, that is, $\left[t_{k}-\Delta T_{\mathrm{DB}}, t_{k}\right]$, where $\Delta T_{\mathrm{DB}}$ is the width of the temporal window. In general, $\Delta T_{\mathrm{DB}}$ is set equal to position update time step $\Delta \mathrm{T}_{p}$, so that all the observations are used only once. It is worth mentioning that $\Delta T_{\mathrm{DB}}$ could be larger or smaller than $\Delta \mathrm{T}_{p}$. Sometimes, there may be not enough RSSI observations for an unknown node in low dynamic scenario, and $\Delta T_{\mathrm{DB}}$ is set larger than $\Delta \mathrm{T}_{p}$ in order to use the previously collected measurements. On the contrary, there may be too many RSSI observations for a mobile node in high dynamic scenario, and $\Delta T_{\mathrm{DB}}$ is set smaller than $\Delta \mathrm{T}_{p}$ in order to use the freshest measurements. In principle, $\Delta T_{\mathrm{DB}}$ is chosen, depending on the prior knowledge of mobility degree of the unknown nodes. In practice, it may happen that more than one measurement is available between two WSN nodes at certain times. In this case, a weighted average scheme is applied, and the weight associated to a measurement is calculated according to an exponential function which takes as input the time difference between the current time $t_{k}$ and the time stamp attached to this measurement. In other words, much lower weight is assigned to the old measurement while much higher weight is assigned to the new one. For the multiple detections of RFID devices, however, weighted average is not necessary because only the freshest one is used. It is supposed that the RFID detection is exceedingly reliable and the old detection event can be neglected.

3. Position estimation. In this step the hybrid location engine estimates the positions of mobile nodes by using location information and measurements which are provided by the previous two steps. Moreover, a cooperation scheme is applied where the location engine, apart from RSSI measurements from anchors, uses also RSSI measurements performed between mobile nodes, since two mobile WSN nodes are able to communicate with each other and to perform corresponding RSSI observations. The adoption of cooperation improves not only the positioning accuracy but also system robustness (i.e., position estimation availability), as more measurements are available to localize the mobile nodes. Nevertheless, the cooperation can be merely applicable to mobile nodes equipped with WSN devices, because both UHF-RFID tag and HF badge are passive devices and cannot communicate with other passive devices for range or range-related observations. Since the HF badge can be detected by the reader in a very short distance (e.g., a few centimeters), this badge detection event can be seen as quite accurate localization information. In principle, whenever an HF badge is detected, the estimated position of the associated mobile node is set to the HF reader's position, and other observations (e.g., RSSI or UHF-RFID detections) are ignored. Since the HF badge readers are only installed at the door, mainly for the purpose of access control, they provide only sporadic detection events. In most of the time, the hybrid location engine relies on RSSI measurements from WSN devices and UHF-RFID events for localization. In order to have a good estimate of a mobile's position, the location engine adopts a hybrid cooperative tracking algorithm, namely hybrid cooperative extended Kalman filter (hcEKF), which takes into account all the available observations, that is, RSSI measurements performed between WSN nodes (i.e., WSN mobiles to WSN anchors or WSN mobiles to WSN mobiles) and UHF-RFID tag detection events. More details of the adopted hcEKF is presented in Section 4. At the end of the estimation process, all the estimated positions are displayed on the map and are uploaded to the $\mathrm{DB}$ with a time stamp.

The periodic repetitions of these three steps form the whole procedure of the hybrid location engine, which can be summarized as pseudo code as Algorithm 1 .

\section{Hybrid cooperative positioning algorithm}

The implemented hybrid cooperative positioning algorithm is based on Kalman filter (KF), which is an efficient and recursive estimator for discrete time linear filtering problem [55]. There are many extensions and generations of KF, and here the standard extended KF (EKF) is adopted due to its simplicity. Here the formulation of EKF is simply introduced in order to have a better understanding of the proposed positioning system.

\subsection{EKF introduction}

EKF is a simple extension of KF for nonlinear problems [55] and is widely applied in navigation and tracking systems. In principle, EKF includes two phases: prediction phase, during which the system state is estimated based on system behaviors, and update phase, during which the system state is corrected by using the available observations. 


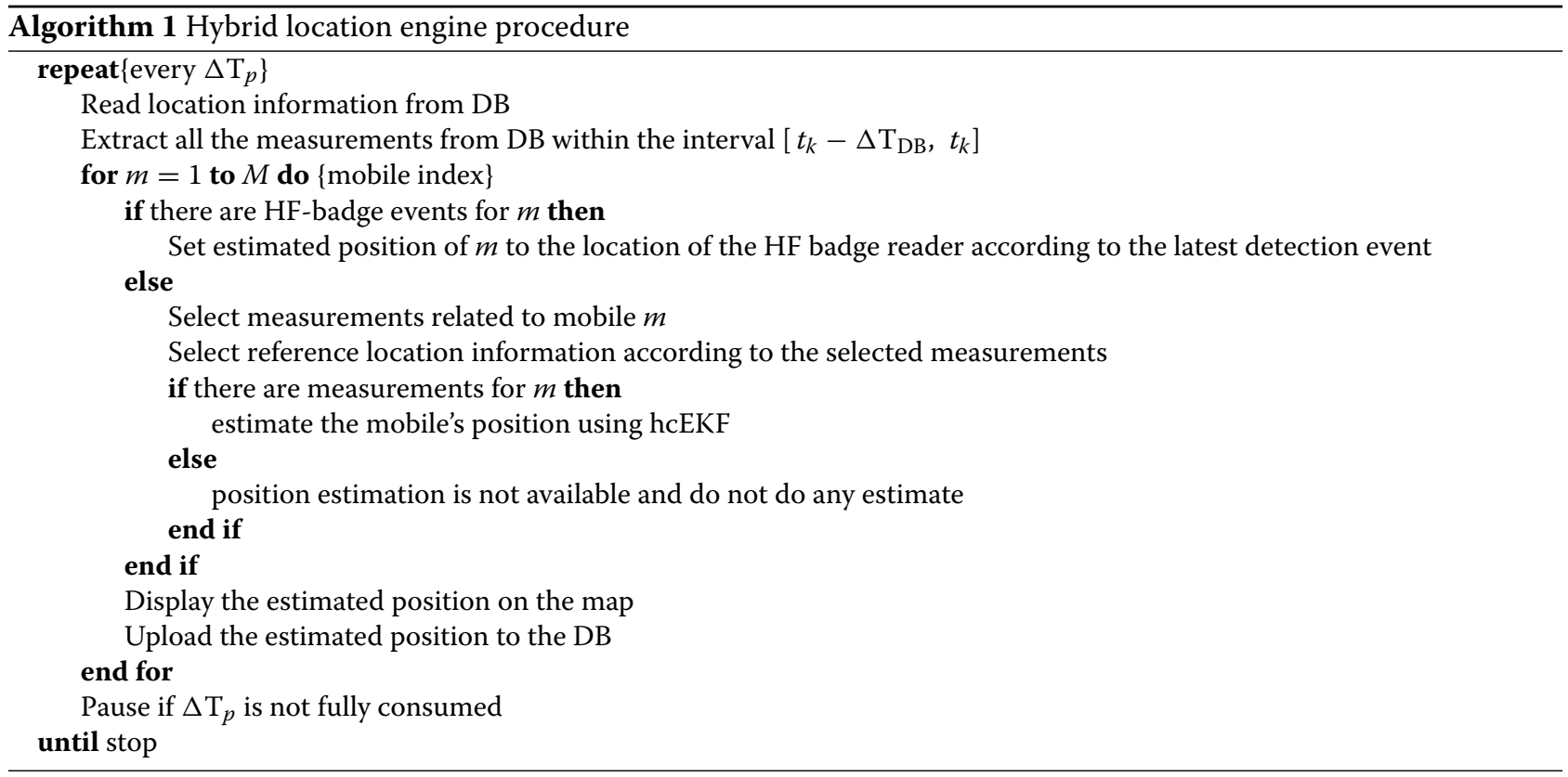

\subsubsection{Prediction}

In prediction phase, the current a priori estimates of state $\hat{\mathbf{x}}_{k \mid k-1}$ and of error covariance $\mathbf{P}_{k \mid k-1}$ are drawn from the previous a posteriori ones of state $\hat{\mathbf{x}}_{k-1 \mid k-1}$ and of error covariance $\mathbf{P}_{k-1 \mid k-1}$ by using the following two equations:

$$
\begin{aligned}
& \hat{\mathbf{x}}_{k \mid k-1}=f\left(\hat{\mathbf{x}}_{k-1 \mid k-1}, \mathbf{w}_{k-1}\right), \\
& \mathbf{P}_{k \mid k-1}=\mathbf{A}_{k} \mathbf{P}_{k-1 \mid k-1} \mathbf{A}_{k}^{T}+\mathbf{Q}_{k-1} .
\end{aligned}
$$

where $f$ is the state transition function and $\mathbf{A}_{k}=$ $\left.\frac{\partial f}{\partial \mathbf{x}}\right|_{\hat{\mathbf{x}}_{k-1 \mid k-1}}$ is the corresponding Jacobian matrix calculated at the previous a posteriori state estimate $\hat{\mathbf{x}}_{k-1 \mid k-1}$. $\mathbf{w}_{k-1}$ is the process noise and is assumed as Gaussian distributed with covariance $\mathbf{Q}_{k}$, that is, $\mathbf{w}_{k-1} \sim \mathcal{N}\left(0, \mathbf{Q}_{k-1}\right)$.

\subsubsection{Update}

In update phase, the $a$ priori estimates $\left(\hat{\mathbf{x}}_{k \mid k-1}\right.$ and $\left.\mathbf{P}_{k \mid k-1}\right)$ are corrected the a posteriori estimates $\left(\hat{\mathbf{x}}_{k \mid k}\right.$ and $\left.\mathbf{P}_{k \mid k}\right)$ by using the available measurements $\mathbf{z}_{k}$. In more detail, innovation vector $\tilde{\mathbf{y}}_{k}$ and optimal Kalman gain $\mathbf{K}_{k}$ are computed as (3) and (4), respectively, as follows:

$$
\begin{aligned}
& \tilde{\mathbf{y}}_{k}=\mathbf{z}_{k}-h\left(\hat{\mathbf{x}}_{k \mid k-1}, \mathbf{v}_{k}\right), \\
& \mathbf{K}_{k}=\mathbf{P}_{k \mid k-1} \mathbf{H}_{k}^{T}\left(\mathbf{H}_{k} \mathbf{P}_{k \mid k-1} \mathbf{H}_{k}^{T}+\mathbf{R}_{k}\right)^{-1},
\end{aligned}
$$

where $h$ is the observation function and $\mathbf{H}_{k}=\left.\frac{\partial h}{\partial \mathbf{x}}\right|_{\hat{\mathbf{x}}_{k \mid k-1}}$ is the corresponding Jacobian matrix evaluated around the a priori state estimate $\hat{\mathbf{x}}_{k \mid k-1}$. $\mathbf{v}_{k}$ is the measurement noise and is also assumed as Gaussian distributed with covariance $\mathbf{R}_{k}$, that is, $\mathbf{v}_{k} \sim \mathcal{N}\left(0, \mathbf{R}_{k}\right)$.
After that, the a posteriori estimates are obtained as follows:

$$
\begin{aligned}
& \hat{\mathbf{x}}_{k \mid k}=\hat{\mathbf{x}}_{k \mid k-1}+\mathbf{K}_{k} \tilde{\mathbf{y}}_{k}, \\
& \mathbf{P}_{k \mid k}=\left(\boldsymbol{I}_{n}-\mathbf{K}_{k} \mathbf{H}_{k}\right) \mathbf{P}_{k \mid k-1} .
\end{aligned}
$$

The recursive computation of Equations 1 to 6 makes up the EKF solutions of a dynamic system.

\subsection{Measurement modeling}

The available measurements are related to the distance between two RF devices using different models, which are adopted in hcEKF.

\subsubsection{WSN measurement model}

The RSSI measurements performed among WSN nodes are linked to the distance observation by adopting the log-normal shadowing path loss model [56], where the received power $\tilde{P}$ (expressed in $\mathrm{dBm}$ ) is seen as a logarithmic function of the distance ( $d$ in meters) between the transmitter and the receiver:

$$
\tilde{P}(d)=P_{0}-10 \alpha \log _{10}\left(d / d_{0}\right)+X_{\sigma},
$$

where $P_{0}$ (expressed in $\mathrm{dBm}$ ) is the mean power received at the reference distance $d_{0}$ (typically $1 \mathrm{~m}$ ), $\alpha$ is the path loss exponent, and $X_{\sigma}$ is an additive measurement noise. For simplicity, $X_{\sigma}$ is assumed to be Gaussian distributed with zero mean and variance $\sigma_{\mathrm{dB}}^{2}$, that is, $X_{\sigma} \sim$ $\mathcal{N}\left(0, \sigma_{\mathrm{dB}}^{2}\right)$. This model only considers the path loss of RF signal and does not takes into account multipath or any other effects. It is worth reminding that these parameters depend greatly on the environment and the operating frequency. Calibrations are required before applying this model. 


\subsubsection{UHF-RFID measurement model}

Concerning the UHF-RFID measurements, as in [11], each detection event is translated to a distance measurement equal to half of the reader interrogation range, $r$. In other words, the UHF-RFID detection is seen as constant distance measurement,

$$
\tilde{d}=r / 2+n,
$$

where $n$ is the measurement noise, and it is hard to know the exact distribution of this noise. Here we assume it satisfies Gaussian distribution with zero mean and a variance depending on the radio coverage $r$. This assumption may be not true but it is suitable for EKF to use the UHF-RFID observations. It is worth reminding that the RFID detection is treated as distance measurement equal to $r / 2$, and this measurement is always positive.

\subsection{Hybrid cooperative EKF}

The adopted hybrid cooperative EKF (hcEKF) is first proposed in [12], and it adds hybrid and cooperative features onto the standard EKF. In principle, the hcEKF algorithm is divided into three parts: state modeling, hybridization, and cooperation, which are introduced in the following.

\subsubsection{State modeling}

The positioning complexity strongly depends on the modeling of the system dynamics, and in this algorithm we choose the system state which is the position of unknown mobiles, that is, $\hat{\mathbf{x}}_{k}=\left[\hat{x}_{k}, \hat{y}_{k}\right]$. Here only $2 \mathrm{D}$ localization is considered but the extension to $3 \mathrm{D}$ case is straightforward.

According to the this model, the state transition function $f$ is a linear function of the state:

$$
\hat{\mathbf{x}}_{k \mid k-1}=f\left(\hat{\mathbf{x}}_{k-1 \mid k-1}, \mathbf{w}_{k-1}\right)=\hat{\mathbf{x}}_{k-1 \mid k-1}+\mathbf{w}_{k-1} .
$$

In this case, the process noise $\mathbf{w}_{k-1}$ models the unknown movements along $x$ and $y$ axes. We let $\Delta t_{k}$ denote the time difference between $\hat{\mathbf{x}}_{k \mid k-1}$ and $\hat{\mathbf{x}}_{k-1 \mid k-1}$, and the covariance matrix $\mathbf{Q}_{k-1}$ can be expressed as:

$$
\mathbf{Q}_{k-1}=\left[\Delta t_{k} \mathbf{I}_{2}\right] \operatorname{diag}\left(\sigma_{\dot{x}}^{2}, \sigma_{\dot{y}}^{2}\right)\left[\Delta t_{k} \mathbf{I}_{2}\right]^{T} .
$$

where $\mathbf{I}_{2}$ is a $2 \times 2$ identity matrix and $\operatorname{diag}\left(\sigma_{\dot{x}}^{2}, \sigma_{\dot{y}}^{2}\right)$ is a $2 \times 2$ diagonal matrix whose diagonal elements are corresponding to the moving speed, which are the differentials of system state.

\subsubsection{Hybridization}

The art of hybridization is to fuse heterogeneous measurements together and to build the corresponding observation functions. Let $\mathcal{A}=\{1,2, \ldots A\}, \mathcal{M}=\{1,2, \ldots M\}$, in which $\mathcal{R}=\{1,2, \ldots R\}$ denote the sets of fixed WSN anchors, WSN mobiles, and fixed RFID readers, respectively. For a generic mobile node $m$ at time $k, \mathcal{A}_{k} \subseteq \mathcal{A}$,
$\mathcal{M}_{k} \subseteq \mathcal{M}$, and $\mathcal{R}_{k} \subseteq \mathcal{R}$ denote the subsets of connected devices (WSN anchors, WSN mobiles, and RFID readers). Note that here $m$ is abbreviated for simplicity of denotation.

Therefore, the generic observation vector can be written as

$$
\mathbf{z}_{k}=\left[\begin{array}{lll}
\tilde{\mathbf{P}}_{\mathcal{A}_{k}} & \tilde{\mathbf{P}}_{\mathcal{M}_{k}} & \tilde{\mathbf{d}}_{\mathcal{R}_{k}}
\end{array}\right]^{T}
$$

where $\tilde{\mathbf{P}}_{\mathcal{A}_{k}}$ and $\tilde{\mathbf{P}}_{\mathcal{M}_{k}}$ denote the sets of RSSI measures from WSNs, while $\tilde{\mathbf{d}}_{\mathcal{R}_{k}}$ denotes the set of RFID-based distance measurements. Note that the RSSI is not transformed into distance measurement and is directly used to feed the positioning algorithm, because the assumption of Gaussian measurement errors does not hold for RSS-based distance measurements [57].

For the a priori estimate $\hat{\mathbf{x}}_{k \mid k-1}$, the corresponding observation function could be one of the three forms

$h\left(\hat{\mathbf{x}}_{k \mid k-1}\right) \in\left[h_{\mathcal{A}_{k}}\left(\hat{\mathbf{x}}_{k \mid k-1}\right) h_{\mathcal{M}_{k}}\left(\hat{\mathbf{x}}_{k \mid k-1}\right) h_{\mathcal{R}_{k}}\left(\hat{\mathbf{x}}_{k \mid k-1}\right)\right]$,

where $h_{\mathcal{A}_{k}}\left(\hat{\mathbf{x}}_{k \mid k-1}\right), h_{\mathcal{M}_{k}}\left(\hat{\mathbf{x}}_{k \mid k-1}\right)$, and $h_{\mathcal{R}_{k}}\left(\hat{\mathbf{x}}_{k \mid k-1}\right)$ are the relative observation functions, referring to the subsets of connected WSN anchors, WSN mobiles, and RFID readers, respectively. More specifically, $h_{\mathcal{A}_{k}}\left(\hat{\mathbf{x}}_{k \mid k-1}\right)$ is calculated by using Equation 7,

$$
h_{\mathcal{A}_{k}}\left(\hat{\mathbf{x}}_{k \mid k-1}\right)=P_{0}-10 \alpha \log _{10}\left(\operatorname{dist}\left(\hat{\mathbf{x}}_{k \mid k-1}, \boldsymbol{p}_{k}^{i}\right) / d_{0}\right) \text {, }
$$

where $\boldsymbol{p}_{k}^{i}\left(i \in \mathcal{A}_{k}\right)$ is the position of $i$ th WSN anchor at time $\mathrm{k}$ and $\operatorname{dist}(\cdot)$ is the operator of the Euclidean distance computation, e.g., $\operatorname{dist}\left(\mathbf{x}_{1}, \mathbf{x}_{2}\right)=$ $\sqrt{\left(x_{1}-x_{2}\right)^{2}+\left(y_{1}-y_{2}\right)^{2}}$.

In Equation 13, $h_{\mathcal{M}_{k}}\left(\hat{\mathbf{x}}_{k \mid k-1}\right)$ is calculated similarly, but the positions of mobile are used instead of those of anchors. Note that the uncertainty of mobile's position is considered on the measurement noise and it is found in Section 4.3.3. In addition, $h_{\mathcal{R}_{k}}\left(\hat{\mathbf{x}}_{k \mid k-1}\right)$ can be computed by using (8)

$$
h_{\mathcal{R}_{k}}\left(\hat{\mathbf{x}}_{k \mid k-1}\right)=\operatorname{dist}\left(\hat{\mathbf{x}}_{k \mid k-1}, \boldsymbol{p}_{k}^{l}\right)
$$

where $\boldsymbol{p}_{k}^{l}\left(l \in \mathcal{R}_{k}\right)$ is the position of $l$ th RFID reader at time $k$. More details about how to set the observation function can be found in [12].

\subsubsection{Cooperation}

Cooperations among mobile nodes increase the signal of opportunities for more range or range-related measurements. Uncertainty about mobile's position, however, should be taken into appropriate considerations. Otherwise, cooperation might do harm to the position estimation, that is, the estimated positions could even diverge 
further away from the real ones than the noncooperative case. Usually, the uncertainty of mobile's position is evaluated as the trace of its error covariance matrix.

In hcEKF this uncertainty is mapped on the RSSI measurement and is modeled as additional additive noise on the RSS measurements. In other words, the measurement noise variance from mobile nodes is as the sum of the intrinsic measurement variance plus a contribution from the neighboring mobile position's uncertainty, i.e.,

$$
\sigma_{\mathcal{M}_{k}^{j}}^{2}=\sigma_{\mathrm{dB}}^{2}+\sigma_{\mathcal{X}_{k}^{j}}^{2},
$$

where $j \in \mathcal{M}_{k}$ is the $j$ th connected mobile nodes and $\sigma_{\mathrm{dB}}^{2}$ is the intrinsic noise variance. Moreover, $\sigma_{\mathcal{X}_{k}^{j}}^{2}$ is the additional noise variance and is a function of the trace of error covariance matrix $\left(\operatorname{trace}\left(\mathbf{P}_{k}^{j}\right)\right)$, which is indicated in more details in [12].

Supposing that all the available measurements are independent with each other, the measurement error covariance matrix $\mathbf{R}_{k}$ is a diagonal matrix given by:

$$
\mathbf{R}_{k}=\operatorname{diag}(\underbrace{\ldots \sigma_{\mathcal{A}_{k}^{i}}^{2} \ldots}_{i \in \mathcal{A}_{k}} \underbrace{\ldots \sigma_{\mathcal{M}_{k}^{j}}^{2} \ldots}_{j \in \mathcal{M}_{k}} \underbrace{\ldots \sigma_{\mathcal{R}_{k}^{l}}^{2} \ldots}_{l \in \mathcal{R}_{k}}) .
$$

In distributed localization systems, cooperations among mobile nodes increase network traffic to transmit the cooperation packets. In this case, however, cooperations are done in the centralized location engine and no network traffic is generated. The whole hcEKF procedure is presented as pseudo code as Algorithm 2.

\subsection{Complexity analysis}

The computational complexity of EKF is mainly upon the matrix inversion and matrix multiplication. For each state estimate, in (4), matrix inversion is computed with asymptotic complexity $\mathcal{O}\left(\mathbb{R}^{3}\right)$ [58], where $\mathbb{R}$ is the dimension of measurement noise covariance $\mathbf{R}$ or the number of available measurements; in (6), matrix multiplication is computed with asymptotic complexity $\mathcal{O}\left(\mathbb{P}^{3}\right)$ [58], where $\mathbb{P}$ is the dimension of error covariance or the dimension of the state vector. In the positioning applications, the number of measurements is usually larger than the dimension of state in order to solve the ambiguity of position estimate. Hence, the complexity of EKF is the computation of inverting matrices in our application. Let $\left|\mathcal{A}_{k}\right|,\left|\mathcal{M}_{k}\right|$, and $\left|\mathcal{R}_{k}\right|$ denote the cardinality of the corresponding sets $\mathcal{A}_{k}$, $\mathcal{M}_{k}$, and $\mathcal{R}_{k}$. The complexity of adopted hcEKF is asymptotically $\mathcal{O}\left(\left(\left|\mathcal{A}_{k}\right|+\left|\mathcal{M}_{k}\right|+\left|\mathcal{R}_{k}\right|\right)^{3}\right)$. For the standard EKF algorithm, the used measurements are only in set $\mathcal{A}_{k}$, and the complexity is asymptotically $\mathcal{O}\left(\left|\mathcal{A}_{k}\right|^{3}\right)$. Therefore, the complexity of hcEKF is increased $\left(1+\frac{\left|\mathcal{M}_{k}\right|+\left|\mathcal{R}_{k}\right|}{\left|\mathcal{A}_{k}\right|}\right)^{3}$ times with respect to standard EKF. For example, suppose that at a specific time, there are two RSSI measures from anchors $\left|\mathcal{A}_{k}\right|=2$, one RSSI measure from mobile node $\left|\mathcal{M}_{k}\right|=1$, and one RFID observation $\left|\mathcal{R}_{k}\right|=1$, the computational complexity of hcEKF is increased about eight times. It is worth reminding that the hcEKF can still localize the mobile node in this case by using the observations from RFID technology.

\section{Simulation and experimental results}

The performance of the proposed tracking system is first evaluated through simulations and then by means of real experiment deployment.

The selected validation scenario is based on the Pervasive Radio Technologies Laboratory at Istituto Superiore Mario Boella (ISMB) and is composed of two adjacent rooms, namely, room 1 and room 2 , which are connected by a corridor (see in Figure 5). This scenario is office environment with building structure mainly composed of metal and the size of it is about $25 \times 12 \mathrm{~m}$. In Figure 5, the blue and the red rectangles inside room 1 and room 2 represent the tables and those at the edges represent the walls, and their material properties are not considered

\section{Algorithm 2 Hybrid cooperative EKF (hcEKF)}

Input: hybrid measurements vector $\mathbf{z}_{k}=\left[\tilde{\mathbf{P}}_{\mathcal{A}_{k}} \tilde{\mathbf{P}}_{\mathcal{M}_{k}} \tilde{\mathbf{d}}_{\mathcal{R}_{k}}\right]^{T}$, the previous a posteriori estimates $\hat{\mathbf{x}}_{k-1 \mid k-1}$ and covariance $\mathbf{P}_{k-1 \mid k-1}$, positioning information $\mathbf{x}_{k}^{j}$ and trace $\left(\mathbf{P}_{k}^{j}\right), \forall j \in \mathcal{M}_{k}$

Output: update the a posteriori estimates $\hat{\mathbf{x}}_{k \mid k}$ and covariance $\mathbf{P}_{k \mid k}$

1: calculate noise covariance for mobile $\sigma_{\mathcal{M}_{k}^{j}}^{2}$ as (15), $\forall j \in \mathcal{M}_{k}$

2: update noise covariance $\mathbf{R}_{k}$ using (16)

3: predict state $\hat{\mathbf{x}}_{k \mid k-1}$ as (1)

4: predict error covariance matrix $\mathbf{P}_{k \mid k-1}$ as (2)

5: compute innovation $\tilde{\mathbf{y}}_{k}$ as (3)

6: compute Kalman gain $\mathbf{K}_{k}$ as (4)

7: update state $\hat{\mathbf{x}}_{k \mid k}$ using (5)

8: update error covariance matrix $\mathbf{P}_{k \mid k}$ using (6)

9: broadcast $\hat{\mathbf{x}}_{k \mid k}$ and trace $\left(\mathbf{P}_{k \mid k}\right)$ to neighbors 


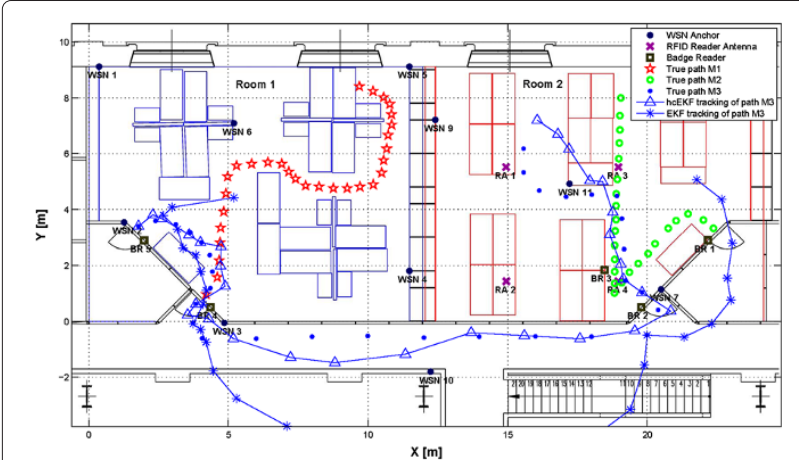

Figure 5 Simulation scenario and the trajectories.

yet, since it is not an easy task. Our work is concentrated on the realization of the hybrid WSN-RFID localization system. These tables and walls are plotted to provide apparent references to the estimated positions.

\subsection{Simulation results}

In the simulation scenario, the following deployment of RF devices is adopted. Eleven WSN anchor nodes (WSN 1 to 11 in Figure 5) are placed around the rooms to optimize the geometry distribution for positioning; four UHF-RFID antennas (RA 1 to 4 in Figure 5) are deployed only in room 2; five badge readers (BA 1 to 5 in Figure 5) are installed at the doors to provide access control; three hybrid mobile nodes are considered; and all of them are equipped with a WSN device, a UHF-RFID tag, and an HF badge.

Three different trajectories are considered, and the three mobile nodes move along them respectively. Figure 5 shows the exact positions of three paths: the first one is in room 1 and is represented by red pentagrams and mobile; the second one is in room 2 and is represented by green circles; the third one connects from room 1 to room 2 through the corridor and is represented by blue dots.

RSSI measurements are generated by using the lognormal model reported in (7). The model parameters are from an experiment carried out in 2009 [59]; in more details, $P_{0}=-49, \alpha=3.3$, and $\sigma_{\mathrm{dB}}=5.5$. The sensitivity of the WSN receiver is set to $-90 \mathrm{dBm}$, which determines the connectivity of two WSN nodes. A badge event is generated by the badge reader when a badge passes through the doors. A tag detection event is provided by the UHFRFID antenna when a passive UHF-RFID tag is within the coverage area, which is modeled as a circle with radius $r=2 \mathrm{~m}$.

One hundred Monte Carlo (MC) simulations are performed to provide steady statistics. The tracking performance is evaluated as root mean square of positioning errors (RMSE) given by:

$$
\text { RMSE }=\sqrt{\frac{1}{N \cdot K} \sum_{i=1}^{N} \sum_{k=1}^{K}\left\|\hat{\mathbf{p}}_{k}^{i}-\mathbf{p}_{k}^{i}\right\|^{2},}
$$

where $N$ is the number of $\mathrm{MC}$ runs and $K$ is the number of positions in each trajectory. In addition, $\hat{\mathbf{p}}_{k}^{i}$ and $\mathbf{p}_{k}^{i}$ denote the corresponding estimated and exact positions of mobile node at $i$ th run and $k$ th position. The distance of two positions, $\left\|\hat{\mathbf{p}}_{k}^{i}-\mathbf{p}_{k}^{i}\right\|$, is also known as the positioning error.

Moreover, four different tracking algorithms are tested for comparison: the hcEKF which uses all the available measurements, the hEKF which uses RSSI from WSN anchors and detection events from RFID, the cEKF which uses only RSSI measures from WSN nodes, and the EKF (noncooperative and nonhybrid) which uses only RSSI measurements from WSN anchors.

Figure 5 shows the tracking result of one realization, where only the estimated positions of hcEKF and EKF related to mobile node M3 are plotted to avoid an overcrowded figure. Thanks to the HF badge detection, the hcEKF is accurately initialized, while the EKF has to be initialized to the coordinates of the scenario's center because it can only use RSSI measures. When M3 is in the corridor, the EKF diverges due to the bad geometry of the WSN anchor deployment while the hcEKF is able to follow the real trajectory thanks to hybridization of RFID detection and the cooperation with the other mobile nodes. When M3 approaches room 2, the standard EKF diverges again while the hcEKF is still able to track the mobile by fusing measurements from HF badge reader and UHF-RFID tag reader.

Figure 6 shows the simulated tracking performance in terms of cumulative distribution function (CDF) and RMSE of the positioning errors. It can be observed that the hcEKF, which fuses hybrid measurements of RSSI

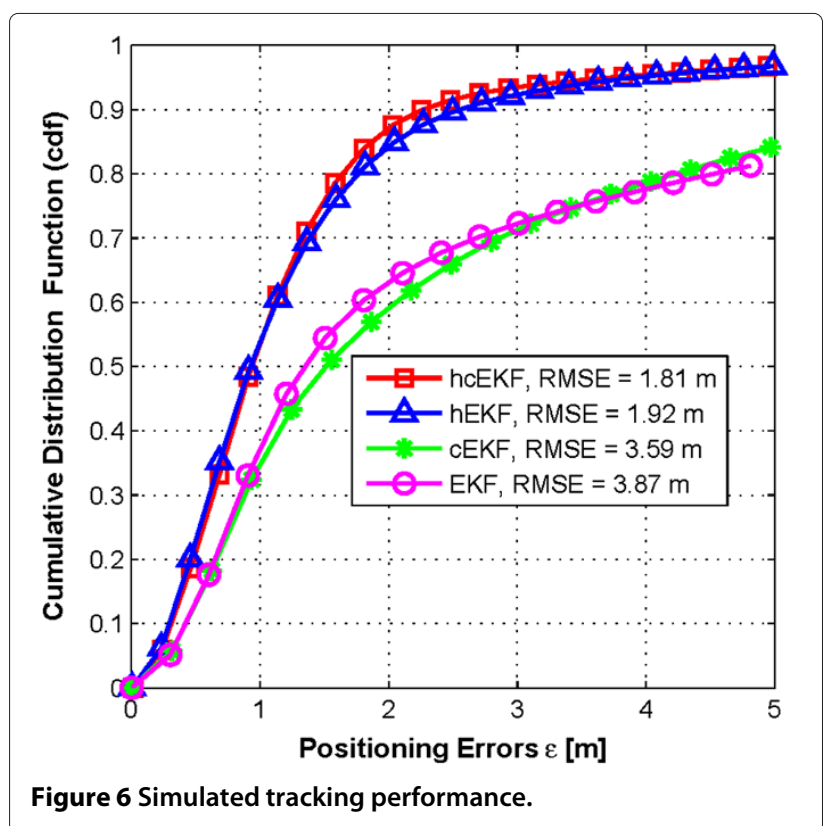



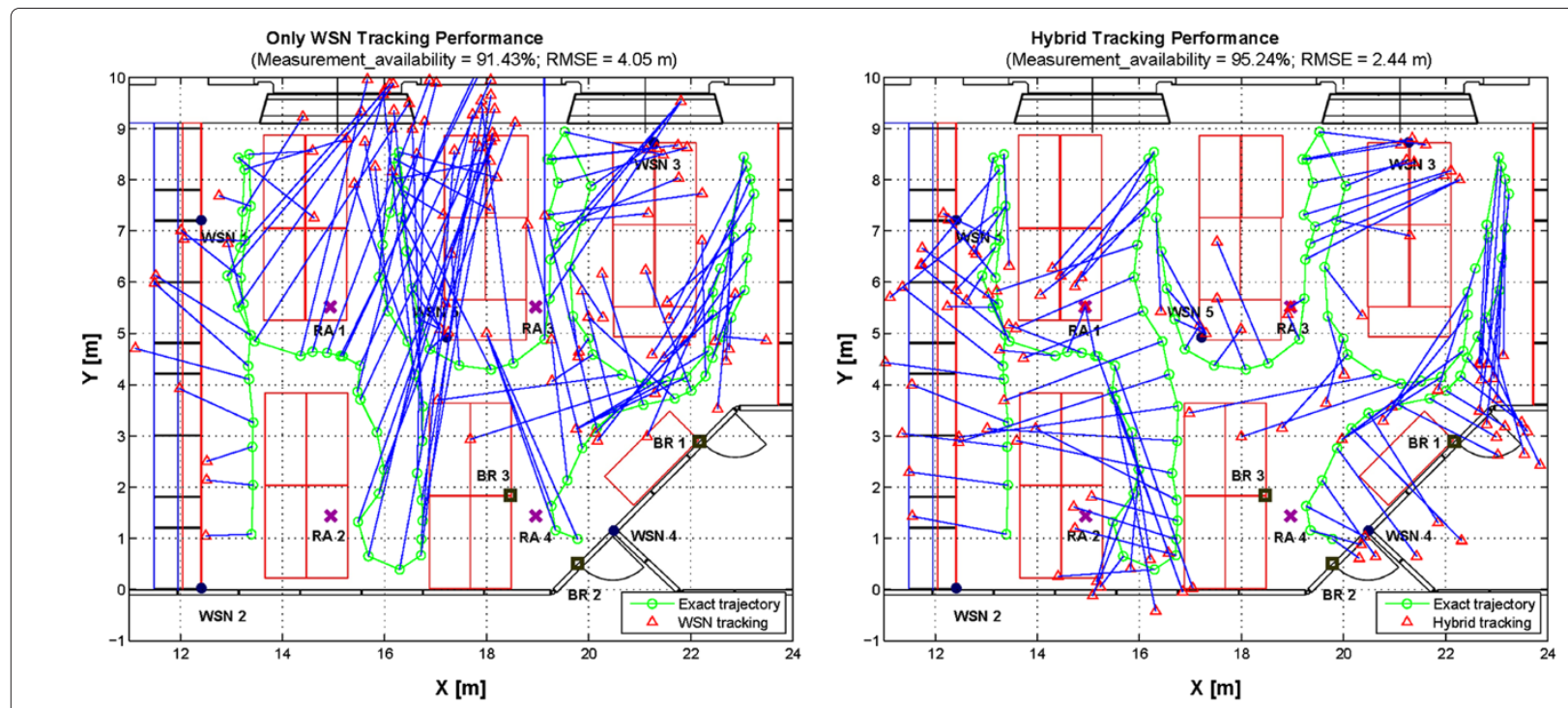

Figure 7 Experimental tracking results.

from WSN and detection events from RFID readers and adopts cooperation among mobile nodes, shows the best tracking performance, i.e., best CDF curve and smallest RMSE. The hEKF outperforms cEKF, which indicates that the integration of RFID technology can overcome the inherent disadvantages of WSN RSSI localization. The cEKF and standard EKF provide similar performance, because there are lots of anchors nodes that provide enough RSSI observations and the gain of cooperation is not obvious. The gain of the adoption RFID is showed by the simulation, and it is difficult to define the numerical gain on the positioning lower bound since heterogeneous measurements are used.

\subsection{Experimental results}

Due to the lack of devices, the availability of WSN devices was not sufficient to allow a full deployment as the simulation. The experiment was carried out only in room 2 , and the RF devices were only deployed in room 2 as Figure 7. In total, five WSN nodes (WSN 1 to 5), four UHF-RFID antennas (RA 1 to 4), and three HF badge readers (BA 1 to 3 ) were deployed. A mobile equipped with the previously mentioned RF devices did a pedestrian movement along a zigzag trajectory in the experimental area.

Before tracking the mobile, some RSSI measurements are used to calibrate the log-normal model in (7). The relative results are shown in Figure 8 . Based on these measurements, the model parameters is chosen as $P_{0}=$ $-50.8, \alpha=1.3$, and $\sigma_{\mathrm{dB}}=6.1$. These parameters indicate that the environment is harsh and the RSSI measurements is quite noisy, posing a challenge for tracking.

The final experimental results are presented in Figure 7, where the left part shows the tracking result of only WSN measurements and the right part shows that of hybrid tracking. Since the RSSI measurements contained large noise, we adopt an optimization method that corrects the bad position estimate to the position of RFID reader when RFID detection is available. Moreover, the measurement availability and RMSE are reported in the upper part.

Due to the large noise on the RSSI measurements, the tracking trajectory has large errors and the performance is worse than the simulation. By fusing the measurements from RFID technology, the hybrid tracking algorithm is able to track better the maneuvers of mobile, which is consistent with the simulation result. Due to the high packet loss rate, sometimes there is no RSSI measurement to

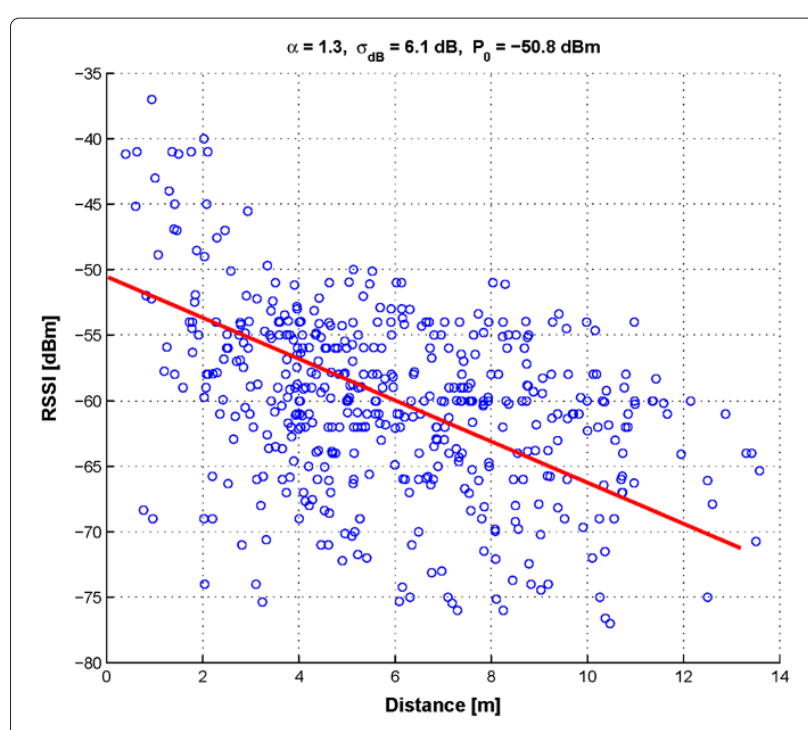

Figure 8 Channel model based on RSSI values. 
be used to track the mobile, and the observation from RFID can improve system availability. The adoption of hybridization provides improvement of $1.6 \mathrm{~m}$ in RMSE and of $4 \%$ in availability.

\section{Conclusions}

This work presented a hybrid WSN-RFID system for tracking people and objects in indoor scenarios. The joint use of heterogeneous technologies can overcome the limitations of each other: WSN system provides adequate RSSI observations but with large errors, and RFID system provides accurate detections but with sparse observations. Thanks to the hybridization of RFID measurements and cooperation among mobile nodes, the proposed positioning solution based on EKF is able to increase the robustness and accuracy of indoor positioning systems in harsh propagation conditions. Simulation and experimental results showed that the hybrid WSN-RFID configuration outperformed the set-ups employing single technology. Therefore, we can conclude that indoor positioning systems can effectively benefit from hybrid WSN and RFID technologies. Furthermore, the proposed configuration is cost-effective in situations where WSN and RFID devices are already deployed for other purposes such as environment monitoring or access control.

\section{Endnote}

${ }^{\mathrm{a}}$ This article is an extended version of a conference paper [13] published at 'The fourth International EURASIP Workshop on RFID Technology'.

\section{Competing interests}

The authors declare that they have no competing interests.

\section{Acknowledgements}

This paper has been partially supported by regional project 'GA-RF-WSN' and by the European FP7 project BUTLER, under contract no. 287901.

Received: 21 November 2012 Accepted: 26 March 2013

Published: 22 April 2013

\section{References}

1. O Vermesan, P Friess, P Guillemin, S Gusmeroli, H Sundmaeker, A Bassi, IS Jubert, M Mazura, M Harrison, M Eisenhauer, P Doody, Internet of Things strategic research roadmap. Technical report, The loT European Research Cluster - European Research Cluster on the Internet of Things - IERC (2009)

2. G Chen, D Kotz, A survey of context-aware mobile computing research. Dartmouth Computer Science Technical Report TR2000-381, Darmouth College (2000)

3. IF Akyildiz, W Su, Y Sankarasubramaniam, E Cayirci, Wireless sensor networks: a survey. Comput. Netw. 38(4), 393-422 (2002)

4. SA Munir, B Ren, W Jiao, B Wang, D Xie, J Ma, in Proceedings of the 21st International Conference on Advanced Information Networking and Applications Workshops, Washington, DC, May 2007, vol. 2. Mobile wireless sensor network: architecture and enabling technologies for ubiquitous computing (IEEE Computer Society, Washington, 2007), pp. 113-120

5. B Nath, F Reynolds, R Want, RFID technology and applications. Pervasive Comput. IEEE. 5(1), 22-24 (2006)
6. E Welbourne, L Battle, G Cole, K Gould, K Rector, S Raymer, M Balazinska, G Borriello, Building the Internet of Things using RFID: the RFID ecosystem experience. Internet Computing, IEEE. 13(3), 48-55 (2009)

7. J Sung, T Sanchez Lopez, D Kim, in Fifth Annual IEEE International Conference on Pervasive Computing and Communications Workshops. The EPC sensor network for RFID and WSN integration infrastructure. White Plains, 19-23 March 2007, pp. 618-621

8. C Ma, Y Wang, G Ying, in 2011 Fourth International Conference on Information and Computing. The pig breeding management system based on RFID and WSN. Shanghai, 25-27 April 2011, pp.30-33

9. L Guo, W Fang, G Wang, L Zheng, in 2010 International Conference on Computer and Communication Technologies in Agriculture Engineering Intelligent traffic management system base on WSN and RFID. Chengdu, 12-13 June 2010, pp. 227-230

10. S Long, R Kooper, GD Abowd, CG Atkeson, in Proceedings of the 2nd Annual International Conference on Mobile Computing and Networking, MobiCom96. Rapid prototyping of mobile context-aware applications: the cyberguide case study 2 scenarios for a mobile context-aware application. White Plains, NY, 11-12 November 1996 (ACM Press, New York, 1996), pp. 97-107

11. Z Xiong, F Sottile, MA Spirito, R Garello, in 2011 4th IFIP International Conference on New Technologies Mobility and Security. Hybrid indoor positioning approaches based on WSN and RFID. Paris, 7-10 February 2011, pp. 1-5.

12. Z Xiong, F Sottile, MA Caceres, MA Spirito, R Garello, in in 2011 IEEE-APS Topical Conference on Antennas and Propagation in Wireless Communications (APWC). Hybrid WSN-RFID cooperative positioning based on extended Kalman filter (IEEE Conference Publications, Piscataway, 2011), pp. 990-993

13. X Zhoubing, S Zhen Yu, A Scalera, F Sottile, R Tomasi, MA Spirito, in 2012 Fourth International EURASIP Workshop on RFID Technology (EURASIP RFID) Enhancing WSN-based indoor positioning and tracking through RFID technology. Torino, 27-28 September 2012, pp. 107-114

14. J Hightower, G Borriello, A survey and taxonomy of location systems for ubiquitous computing. Technical Report UW-CSE 01-08-03, University of Washington, Computer Science and Engineering (2001)

15. J Hightower, G Borriello, Location systems for ubiquitous computing Computer. 34(8), 57-66 (2001)

16. R Want, A Hopper, V Falcão, J Gibbons, The active badge location system. ACM Trans. Inf. Syst. 10(1), 91-102 (1992)

17. P Bahl, VN Padmanabhan, Radar: an in-building RF-based user location and tracking system (2000), in Proceedings of INFOCOM 2010. Nineteenth Annual Joint Conference of the IEEE Computer and Communications Societies, Victoria Conference Centre, March 2010, vol. 2 (IEEE Conference Publications, Piscataway, 2010), pp. 775 -784

18. LM Ni, Y Liu, Y Cho Lau, AP Patil, LANDMARC: indoor location sensing using active RFID Wireless Netw. 10, 701-710 (2004). doi:10.1023/B:WINE.0000044029.06344.dd

19. M Vossiek, L Wiebking, P Gulden, J Wiehardt, C Hoffmann, P Heide, Wireless local positioning. IEEE Microwave Mag. 4(4), 77-86 (2003)

20. Iso/IEC 24730-1:2006. (ISO, 2006). http://www.iso.org. Accessed 15 Feb 2006

21. H Liu, H Darabi, P Banerjee, I Liu, Survey of wireless indoor positioning techniques and systems. IEEE Trans. Syst. Man Cyber. 37(6), 1067-1080 (2007)

22. D Niculescu, B Nath, in Proceedings of the 10th annual international conference on Mobile computing and networking, MobiCom 2004. VOR base stations for indoor 802.11 positioning. Taormina, 26 September to 1 October 2004, (ACM, New York, 2004), pp. 58-69

23. K Lorincz, M Welsh, MoteTrack: A robust, decentralized approach to RF-based location tracking. Location-and Context-Awareness. (Springer, Berlin Heidelberg, 2005), pp. 63-82

24. B Eissfeller, D Gänsch, S Müller, A Teuber, in Proceedings of the 17th International Technical Meeting of the Satellite Division of The Institute of Navigation (ION GNSS 2004). Indoor positioning using wireless LAN radio signals. Long Beach Convention Center, Long Beach, 21-24 September 2004

25. W Tranter, K Shanmugan, T Rappaport, K Kosbar, Principles of Communication Systems Simulation with Wireless Applications, 1 st edn. (Prentice Hall Press, Upper Saddle River, 2003) 
26. Skyhook location technology(Skyhook, 2012). (Skyhook, 2012), http:// www.skyhookwireless.com. Accessed 2012

27. KC Cheung, SS Intille, K Larson, An inexpensive Bluetooth-based indoor positioning hack. http://architecture.mit.edu/house_n/documents/ CheungIntilleLarson2006.pdf. Accessed 2006

28. L Aalto, N Göthlin, J Korhonen, T Ojala, in Proceedings of the 2nd International Conference on Mobile Systems, Applications, and Services, MobiSys 2004. Bluetooth and WAP push based location-aware mobile advertising system, ACM, New York, 2004), pp. 49-58

29. MS Bargh, R de Groote, in Proceedings of the First ACM international Workshop on Mobile Entity Localization and Tracking in GPS-less Environments MELT'08. Indoor localization based on response rate of Bluetooth inquiries (ACM, New York, 2008), pp. 49-54

30. ZONITH Indoor Positioning System (ZONITH, 2012). http://www.zonith. com/products/ips. Accessed 2012

31. H Kroandll, C Steiner, in 2010 International Conference on Indoor Positioning and Indoor Navigation (IPIN). Indoor ultra-wideband location fingerprinting. ETH Zurich, Zurich, 15-17 September 2010, pp. 1-5

32. SWang, A Waadt, A Burnic, D Xu, C Kocks, GH Bruck, P Jung, in 7th International Symposium on Wireless Communication Systems (ISWCS), 2010. System implementation study on RSSI based positioning in UWB networks. The University of York, 19-22 September 2010, pp. 36-40

33. Zebra enterprise solutions (Zebra, 2012). http://zes.zebra.com/. Accessed 2012

34. Ubisense Real-time Location Systems Ubisense, 2012. http://www. ubisense.net. Accessed 2012

35. S Tadakamadla, Indoor local positioning system for ZigBee based on RSSI , Thesis. Mid Sweden University, 50 (2006)

36. JM Lopez Garde, I Larranaga, L Muguira, II Vazquez, in Proceedings of the 2010 International Conference on Indoor Positioning and Indoor Navigation (IPIN). An environment adaptive ZigBee-based indoor positioning algorithm. ETH Zurich, Zurich, 15-17 September 2010

37. My-bodyguard (smartTracker, 2012). http://smarttracker.tellu.no/? portfolio-item=my-bodyguard. Accessed 2012

38. A Povalac, J Sandebesta, in 2010 Conference Proceedings ICECom. Phase of arrival ranging method for UHF RFID tags using instantaneous frequency measurement. Dubrovnik, Croatia, 2023 September 2010, (IEEE Press, Piscataway, 2010), pp. 1-4

39. PV Nikitin, R Martinez, S Ramamurthy, H Leland, G Spiess, KVS Rao, in 2010 IEEE International Conference on RFID. Phase based spatial identification of UHF RFID tags. Orange County Convention Center, 14-16 April 2010, pp. 102-109

40. D Arnitz, K Witrisal, U Muehlmann, Multifrequency continuous-wave radar approach to ranging in passive UHF RFID. IEEE Trans. Microw. Theory Techno. 57(5), 1398-1405 (2009)

41. H Don Chon, S Jun, H Jung, S Won, An Using RFID for accurate positioning. J. Global Pos. Syst. (2004). 3(1), 32-39 (2005)

42. SL Ting, SK Kwok, AHC Tsang, GTS Ho, The study on using passive RFID tags for indoor positioning. Int. J. Eng. Bus. Manage. 3(1), 9-15 (2011)

43. M Bouet, AL dos Santos, RFID tags: positioning principles and localization techniques, in 1st IFIP Wireless Days, 2008. Dubai, 24-27 November 2008, pp. 1-5

44. CWang, H Wu, N-F Tzeng, in 26th IEEE International Conference on Computer Communications, INFOCOM 2007. RFID-based 3-D positioning schemes. Anchorage, 6-12 May 2007, 1235-1243

45. M Zhu, G Retscher, K Zhang, Integrated algorithms for RFID-based multi-sensor indoor/outdoor positioning solutions. Geospatial Sci. 22 451-465 (2011)

46. G Retscher, Q Fu, Integration of RFID, GNSS and DR for ubiquitous positioning in pedestrian navigation. 6(1), 56-64 (2007)

47. F Seco, C Plagemann, AR Jimee, W Burgard, in 2010 International Conference on Indoor Positioning and Indoor Navigation (IPIN). Improving RFID-based indoor positioning accuracy using Gaussian processes. ETH Zurich, Zurich, 15-17 September 2010, pp. 1-8

48. Kimaldi (Kimaldi, 2012). http://www.kimaldi.com/. Accessed 2012

49. D Daly, T Melia, G Baldwin, in 2010 International Conference on Indoor Positioning and Indoor Navigation (IPIN). Concrete embedded RFID for way-point positioning' andnez. ETH Zurich, Zurich, 15-17 September 2010, pp. 1-10
50. M Kiers, E Krajnc, M Dornhofer, W Bischof, Evaluation and improvements of an RFID based indoor navigation system for visually impaired and blind people. (IPIN, 2011), http://ipin2011.dsi.uminho.pt/PDFs/Shortpaper/ 44_Short_Paper.pdf. Accessed 2011

51. J Peng, M Zhu, K Zhang, in 2011 International Conference on Indoor Positioning and Indoor Navigation (IPIN). New algorithms based on sigma point Kalman filter technique for multi-sensor integrated RFID indoor/outdoor positioning. Guimaraes, Portugal, 21-23 September 2011, $21-23$

52. Telos rev.B datasheet (Crossbow Technology, 2012). http://bullseye.xbow. com:81/Products/Product_pdf_files/Wireless_pdf/TelosB_Datasheet.pdf. Accessed July 2012

53. STM32W datasheet (ST Microelectronics, 2012). http://www.st.com/ internet/mcu/subclass/1377.jsp. Accessed July 2012

54. A Dunkels, B Gronvall, T Voigt, in 29th Annual IEEE International Conference on Local Computer Networks, 2004. Contiki - a lightweight and flexible operating system for tiny networked sensors. Tampa, 16-18 November 2004 (IEEE Computer Society Press, Los Alamitos, 2004), pp. 455-462

55. G Welch, G Bishop, An introduction to the Kalman filter. Technical Report Chapel Hill, NC 27599-3175. University of North Carolina at Chapel Hill (2006)

56. S Rao, Estimating the ZigBee transmission-range ISM band. EDN. 52(11), 67-74 (2007)

57. N Patwari, A Hero, M Perkins, N Correal, RO'Dea, Relative location estimation in wireless sensor networks. Signal Process Mag. 8(51), 2137-2148 (2003)

58. Computational complexity of mathematical operations (Wikipedia, 2012). http://en.wikipedia.org/wiki/ Computational_complexity_of_mathematical_operations. Accessed 2012

59. D Dardari, F Sottile, WPR.B database: annex of progress report II on advanced localization and positioning techniques: data fusion and applications. Technical Report Deliverable DB.3 Annex 216715, Newcom++ NoE, WPR.B (2009)

doi:10.1186/1687-3963-2013-6

Cite this article as: Xiong et al:: Hybrid WSN and RFID indoor positioning and tracking system. EURASIP Journal on Embedded Systems 2013 2013:6.

\section{Submit your manuscript to a SpringerOpen ${ }^{\circ}$ journal and benefit from:}

- Convenient online submission

- Rigorous peer review

- Immediate publication on acceptance

- Open access: articles freely available online

- High visibility within the field

- Retaining the copyright to your article

Submit your next manuscript at $>$ springeropen.com 